\title{
An immunochemical test for chorionic gonadotrophin
}

\author{
T. D. ORBAN \\ From Sydney, Australia
}

SYNOPSIS An immunochemical procedure for routine laboratory diagnosis of pregnancy utilizing adsorption of human chorionic gonadotrophin onto kaolin and the Ortho pregnancy test reagents is described. The method is suitable for quantitative assay of urinary human chorionic gonado- iv trophin and can be adapted for use with serum. It was found to compare favourably with the $i$ reliability of Galli-Mainini toad tests. It avoids the disadvantages of 'biological' tests and is quicker $\stackrel{+}{\infty}$ to perform.

The long search for tests in vitro for the early diagnosis of pregnancy enters a new phase by the recent discovery of the antigenicity of human chorionic gonadotrophin (H.C.G.) if mixed with a variety of adjuvants. The resultant antibody, usually produced in rabbits, combines with H.C.G. if present in the urine or serum under examination. This combination is made observable by a second reagent which consists of latex particles coated with human chorionic gonadotrophin. In the absence of H.C.G. in the test material the antiserum remains free to combine with the coating and agglutinates the latex. In the presence of adequate amounts of H.C.G. in the test material the antiserum is used up completely and the coated latex added subsequently will remain in smooth suspension.

The advent of commercially available reagents based on these principles must be of interest to workers engaged in the routine laboratory diagnosis of pregnancy. The reagents are marketed as a diagnostic ' $k$ it' by the Ortho Pharmaceutical Corporation whose products were used throughout this study.

The method of using these reagents proposed by the manufacturers consists of mixing urine with the antiserum, incubation of this mixture, addition of coated latex, further incubation, and finally accurate centrifugation $(1,000 \mathrm{~g}$ for two minutes). The manufactuers' instructions stipulate a number of limitations. Urines from patients less than two weeks overdue (under six weeks from the last menstrual period) or after the first trimester, and urines with a specific gravity of less than 1.015 are to be excluded. There is also no mention of serum as the test material. As the Galli-Mainini test is known to give unequivocal answers in a number of instances Received for publication 6 December 1963. falling into the above categories, the refusal to perform the test will be unacceptable to a number of laboratories aiming at the early diagnosis of pregnancy.

These limitations arise not out of any inadequacies of the reagents themselves but rather out of their proposed use. The final reading of the results depends on the clearing or otherwise of the reaction mixture by centrifugation. This step is based on a critical small difference between the specific gravity of the latex and its suspending medium so that only agglutinated latex will be deposited by the specified conditions of centrifugation. As the urine specimens which differ widely in their specific gravity make up one quarter of the final reaction mixture this may lead to false readings. In order to achieve adequate sedimentation of particles with urines of high specific gravity but containing no H.C.G., the antiserum as $\exists$ issued is of sufficient potency to agglutinate the $\delta$ latex into comparatively large aggregates. This in turn leads to the requirements of large amounts of $\mathrm{O}$ H.C.G. in the sample for complete neutralization and the consequent insensitivity of the test. A further consequence of the above is the rather too frequent occurrence of final mixtures of intermediate $\sigma$ turbidity.

The present study is based on methods using $N$ partial purification of H.C.G. and a completely 0 different procedure for reading the results. It is suggested that the method evolved is unequivocal in $\stackrel{\circ}{=}$ interpretation and less time consuming. It is more $\stackrel{\odot}{\sim}$ sensitive that the Galli-Mainini test with which it was compared. It also lends itself to the use of serum and to the quantitative determination of H.C.G. concentration in cases of chorion epithelioma, $\frac{\AA}{\mathbb{D}}$ hydatidiform mole, and teratomata containing chorionic tissue. 


\section{REAGENTS AND METHODS}

PRINCIPLE The H.C.G. contained in the urine sample is adsorbed onto kaolin which is then washed. The hormone is eluted, and the eluate tested immunologically by a slide agglutination technique. The final reading is based on an agglutination inhibition reaction, i.e., no agglutination indicates the presence of H.C.G. in the test material and agglutination its absence.

The method for serum as test material requires a preliminary protein precipitating step.

TREATMENT OF SAMPLE Chilled urine is cleared by centrifugation or filtration. Thorough chilling of the sample before clearing is desirable as this assists in the removal of urates and mucus. Urates add undesirable bulk to the centrifuged kaolin button. Mucus interferes with the adsorption of H.C.G. by adsorbing onto kaolin, thus blocking its surface.

ADSORPTION OF H.C.G. ONTO KAOLIN The cleared urine is mixed with a suspension of kaolin in citrate buffer. The amount of kaolin is so chosen that it is well in excess of adsorption requirements for amounts of H.C.G. necessary to neutralize completely the antiserum used in the test. It is also not too large so that it packs down to a small button after washing and centrifugation and can be resuspended in a small volume of eluent.

The citrate buffer in the proposed amounts has a double role. It results in an optimal final $p \mathrm{H}$ range of 3.8 to 4.2 of the mixture regardless of the initial $p \mathrm{H}$ of the sample. Strongly ammoniacal urines due to extensive bacterial decomposition constitute very rare exceptions. (Only one out of 500 urines was encountered.) The other function of citrate is to eliminate the effect of a factor found in 2 to $3 \%$ of urines tested with the original method. This factor spontaneously agglutinated the latex reagent within seconds of mixing. This was quite independent of the H.C.G. content of the sample and led to false negative results. The responsible factor was not investigated further, beyond the observation that it required calcium ions as co-factor and its interference could be prevented by the addition of E.D.T.A. or citrate.

The time required for mixing and centrifugation of the sample with the buffered kaolin is sufficient for the adsorption of human chorionic gonadotrophin. This was proven in experiments in which a borderline quantity of H.C.G. was added to male urine and the mixture after adding the buffered kaolin was allowed to stand at $37^{\circ} \mathrm{C}$. or at room temperature for 20,10 , five, and 0 minutes before centrifugation.

CENTRIFUgATION AND WASHING OF KAOLIN Centrifugation of the original kaolin suspension for five minutes in an 'angle' head centrifuge at full speed is sufficient to clear the supernatant completely which is then discarded. The deposit is resuspended in a washing solution and recentrifuged for three minutes in a 'swing out' head centrifuge at full speed, again discarding the supernatant. These times and speeds are sufficient for firm packing of the kaolin and the supernatant can then be decanted by inversion, drainage, and vigorous shaking of the inverted tubes without fear of dislodging the deposits.

ELUTION OF H.C.G. The alkalinity and proportions of the eluent allow for residual water enmeshed in the drained kaolin without drying. Drainage for a few seconds only is sufficient.

The eluent also contains sodium chloride which increases the particle size of agglutinated latex, and results in a clearer distinction between unagglutinated and agglutinated mixtures. The addition of salt also leads to clearer eluates. The time required for complete elution was found to be well within the two minutes given in the method.

IMMUNOLOGICAL TESTING OF ELUATE The antiserum is mixed with an acid buffer whose $p \mathrm{H}$ and strength are so chosen as to render prior neutralization of the alkaline eluate superfluous. The final mixture has a $\mathrm{pH}$ of $7 \cdot 2$ to 7.4. Adequate agglutination of the latex occurs at any $p \mathrm{H}$ between 7 and 8 .

The time required for neutralization of the antiserum has been determined experimentally by adding a borderline quantity of H.C.G. to the buffered antiserum and removing 1 drop from this mixture at frequent intervals. The drops were added to drops of coated latex, mixed and 'rocked' for 12 minutes. One minute of delay was sufficient for complete neutralization, whereas 30 seconds still allowed some antiserum activity. A generous two minutes is given in the method.

After mixing buffered antiserum and eluate on a glass tile the coated latex is added and after 12 minutes of 'rocking' the mixture is examined for agglutination. This time should be adhered to. Agglutination of the latex commences after about seven minutes and the size of the particle increases up to 12 minutes. Incubation at $37^{\circ} \mathrm{C}$. before 'rocking' does not improve results.

THE USE OF SERUM AS TEST MATERIAL The preliminary protein precipitation requires the use of metaphosphoric acid, the efficiency of which depends on adequate chilling of solutions and its slow drop-wise addition with constant mixing. Failure to adhere to these conditions results in incomplete protein precipitation which interferes with the adsorbing efficiency of the kaolin. Aqueous solutions of metaphosphoric acid are known to be unstable. Preparation of fresh reagent at weekly intervals is suggested. The possible usefulness of the reagent after more than one week's storage has not been investigated.

The protein precipitation leaves a supernatant which requires neutralization before the addition of buffered kaolin. This latter is designed to shift the $p \mathrm{H}$ of urines from near neutrality to $p \mathrm{H} \mathrm{4}$. Omission of neutralization as a separate step would require a buffered kaolin reagent of much more alkaline $p \mathrm{H}$. Workers preferring to use serum routinely as the test material might choose to make up such a reagent.

PREPARATION OF REAGENTS

BUFFERED KAOLIN For the stock solution (A) Dissolve 
$155.5 \mathrm{~g}$. citric acid $\left(1 \mathrm{H}_{2} \mathrm{O}\right)$ and $76.5 \mathrm{~g}$. trisodium citrate $\left(2 \mathrm{H}_{2} \mathrm{O}\right)$ in approximately $950 \mathrm{ml}$. of distilled water.

Add $8 \mathrm{~g}$. of best quality (acid-washed) kaolin and make up to 1 litre. Preserve with chloroform and store at room temperature. Before dispensing shake thoroughly to suspend the kaolin, then wait for $\mathbf{1 5}$ to $\mathbf{3 0}$ seconds for the chloroform to settle.

FOR THE WORKING SOLUTION (B) Dilute (A) 1:10 with distilled water. Preserve with chloroform and store at room temperature. Before dispensing, shake thoroughly to suspend the kaolin, then wait for 15 to 30 seconds for the chloroform to settle. The $p \mathrm{H}$ of this solution should be $3 \cdot 6$.

WASHING SOLUTION (C) $0.0001 \mathrm{~N}$ hydrochloric acid in physiological saline.

ALKALINE ELUENT (D) Dilute normal sodium hydroxide $1: 10$ with physiological saline.

PHOSPHATE BUfFer (E) Dissolve 11.36 g. disodium hydrogen phosphate (anhyd.) and $2 \cdot 10 \mathrm{~g}$. potassium dihydrogen phosphate in distilled water and make up to

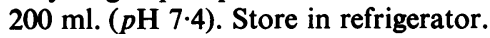

BUFFERED ANTISERUM DILUENT (F) Mix equal parts of $0.2 \mathrm{~N}$ hydrochloric acid and phosphate buffer (E) $(p \mathrm{H} \mathrm{6 \cdot 7)}$. Store in refrigerator.

WORKING ANTISERUM (G) Mix equal parts of Ortho pregnancy test antiserum and $(\mathrm{F})(p \mathrm{H} 6.8)$. Store in refrigerator.

COATED LATEX (H) Use Ortho pregnancy test antigen as issued commercially. Store in refrigerator. This reagent slowly loses potency at room temperature. Dispense small quantities into plastic bottles using one at a time or repeatedly fill a small plastic 'working bottle' from the constantly refrigerated stock.

METAPHOSPHORIC ACID (I) Break off approximately $1 \frac{1}{2}$ in. long stick (about 4 g.) of glacial metaphosphoric acid. Scrape off any white powdery coating and holding the stick with forceps, wash under running water for a few seconds. Dissolve in approximately $50 \mathrm{ml}$. of water with mechanical shaking (15 to 30 minutes). Use this stock solution to make a $0.33 \mathrm{~N}$ working solution by volumetric titration. Store in refrigerator. Stable for one week only.

\section{METHOD FOR URINE}

Mark ' $50 \mathrm{ml}$.' centrifuge tubes, preferably plastic, at 25 and $45 \mathrm{ml}$. with indelible ink or paint.

STEP 1 Fill a tube so marked with cleared urine (centrifuged or filtered while thoroughly chilled) to the first mark. Add (B) to the second mark. Mix thoroughly by inversion. Centrifuge at full speed for five minutes. Decant and discard supernatant.
STEP 2 Add $10 \mathrm{ml}$. of (C) to the deposit. (A $10 \mathrm{ml}$. glass $\overrightarrow{\overrightarrow{\mathrm{C}}}$ dispenser may be used.) Emulsify and suspend the deposit using the base and outside wall of a roundbottomed $15 \mathrm{ml}$. centrifuge tube $\left(4 \frac{1}{2} \times 5 / 8\right.$ in.). Churn $\frac{\overline{\bar{C}}}{\mathrm{D}}$ thoroughly to disperse all clumps. Wipe the outside of $\frac{\bar{s}}{5}$ the smaller tube and pour the suspension into it. Centri- $\stackrel{\otimes}{\circ}$ fuge at full speed for three minutes. Discard supernatant 0 and drain for a few seconds.

STEP 3 Add $0.1 \mathrm{ml}$. of (D) and suspend the deposit by repeated smart tapping with the finger against the lower $\omega^{\circ}$ end of the tube. (A vortex mixer is helpful.) Stand two $\frac{2}{2}$ minutes. Centrifuge at full speed for three minutes.

STEP 4 Tilt the tube toward the horizontal so as to avoid dislodging the deposit and place 1 drop of super- $r$ natant onto a clear glass divided agglutination tile with a $\stackrel{\oplus}{\infty}$ Pasteur pipette. Add 1 drop of $(G)$. (Ensure equal sized 0 drops by using Pasteur pipettes of uniform bore.) Mix with a wooden applicator. Stand two minutes.

STEP 5 Add 1 drop of $(\mathrm{H})$. Mix with an applicator and $\frac{\mathbb{D}}{\mathrm{C}}$ spread out the mixture on the tile to an area of approximately 1 square inch. Slowly 'rock' the tile for 12 minutes. Examine for agglutination of the latex.

\section{METHOD FOR SERUM}

To $6 \mathrm{ml}$. of serum in a conical flask add $12 \mathrm{ml}$. of distilled water and mix. Place the flask into an ice bath within a refrigerator and chill thoroughly. Add drop-wise with constant mixing $3.6 \mathrm{ml}$. of (I). The rate of addition $\frac{\mathrm{D}}{\mathbb{}}$ should not exceed 1 drop/sec. Stand 10 minutes in the 2 ice bath. Centrifuge at full speed for about 10 minutes by $\overrightarrow{\vec{O}}$ pouring the mixture into two $15 \mathrm{ml}$. round-bottomed 3 centrifuge tubes filled equally. Decant supernatants into a single marked ' $50 \mathrm{ml}$.' centrifuge tube as used for urines. Add $3 \mathrm{ml}$. of $0 \cdot 1 \mathrm{~N}$ sodium hydroxide and fill to the first mark with water. Mix and proceed as for urines.

If less than $6 \mathrm{ml}$. of serum is available reduce all: volumes proportionally but still make up to the first 3 . mark $(25 \mathrm{ml}$.) with water.

\section{READING OF RESULTS}

MACROSCOPIC Hold the tile over a black background and 7 illuminate it from below with a shielded lamp at an angle을 so that the light does not shine directly into the observers' N eyes. Agglutinated particles of latex are visible macro- 9 scopically as evenly distributed white granules. Un- $N$ agglutinated particles form a smooth milky suspension.

MICROSCOPIC Examine with low power. Reduce illumination to a minimum. Agglutinated particles are black clumps of varying size. Unagglutinated latex consists of very fine refractile short rod-shaped particles less than ${ }^{+}$ $1 \mu$ in size. Avoid confusion with particles of dirt, accidental contamination with kaolin, fragments of glass, etc. The specific gravity of extraneous matter is very? different from that of latex and collects in the centre of the drop or at its extreme edge after 12 minutes of 'rocking'. Examine the drop about halfway between? 
centre and edge. Microscopic examination is the more reliable.

INTERPRETATION No agglutination = H.C.G. present (pregnant as tested); agglutination = H.C.G. not present (not pregnant as tested).

\section{QUANTITATIVE CONSIDERATIONS}

The quantitative assay of H.C.G. in urine may be of clinical importance in the diagnosis of abnormal chorionic tissue. To this aim, a procedure to depart as little as possible from routine pregnancy testing was adopted which requires identical reagents.

Initial titration of the antibody in the serum supplied in the kit without prior dilution was carried out by serially diluting commercially available H.C.G. of stated potency. Primogonyl (Schering), 1,000 i.u. per ampoule, was used and the stated strength was accepted. When mixed with equal parts of antiserum a concentration of $50 \mathrm{i} . \mathrm{u} . / \mathrm{ml}$. resulted in complete neutralization but $25 \mathrm{i} . \mathrm{u} . / \mathrm{ml}$. resulted in partial agglutination of the subsequently added latex reagent. With this method of detecting endpoints only twofold serial dilutions yielded unequivocal titration results, intermediate concentrations of added H.C.G. showing graded partial agglutinations which were difficult to interpret. Twofold serial dilution was therefore used for all titrations. Mixtures showing the barest trace of agglutination were regarded as the endpoint, whereas an interpolated value was given to series showing sudden strong agglutination after a completely unagglutinated tube.

A dilution of $1+1$ of the antiserum with buffered antiserum diluent reduced the potency as expected by one dilution step without significantly affecting the readability of agglutinations. Repeated checks always resulted in the endpoint being between 25 and $12.5 \mathrm{i} . \mathrm{u}$. $/ \mathrm{ml}$.

The incomplete drying of the washed kaolin deposit and the addition to it of $0.1 \mathrm{ml}$. of alkaline eluent results in an increase of hormone concentration over the original urine of well over one hundredfold. Even allowing for losses inherent in the extraction procedure a one hundredfold increase in concentration can be assumed. This has been checked repeatedly by adding H.C.G. to male urine to a concentration of 1 i.u. $/ \mathrm{ml}$. and processing $25 \mathrm{ml}$. This gives a clear-cut 'positive' test. All subsequent calculations are based on a limiting value of 1 i.u./ml. in urine.

Preliminary titrations of serially diluted eluates showed that the amount of kaolin added with the working buffered kaolin reagent becomes saturated with H.C.G. by concentration somewhat in excess of 10 i.u. $/ \mathrm{ml}$. This is also shown by further addition of an aqueous kaolin suspension $(0.2 \mathrm{ml}$. of a $20 \% \mathrm{w} / \mathrm{v}$ suspension) to the initial supernatant of urines containing H.C.G. in excess of this concentration. This resulted in the further recovery of remaining human chorionic gonadotrophin.

The recommended procedure for quantitative assay is as follows: Commence with three initial urine dilutions: undiluted, 1:10, and 1:100. Proceed with $25 \mathrm{ml}$. of each of these as in 'Method for urine' to the completion of step 3. Set up three rows of small serological agglutin- ation tubes, five in each row, all containing $0.1 \mathrm{ml}$. of alkaline eluent. Tilt the centrifuge tubes containing final eluates to an angle slightly over $90^{\circ}$. Fill $0.1 \mathrm{ml}$. 'blood' pipettes by capillarity with eluates now at the mouths of the centrifuge tubes. Add $0.1 \mathrm{ml}$. to the first agglutination tube in each row, the rows corresponding to the initial urine dilutions. Serially dilute the eluates in each row and proceed as in step 4 .

The final tubes in each row contain an eluate dilution of 1:32 and should show full agglutination in all cases. This is a useful standard of comparison for any partially agglutinated titres. Assuming full saturation of kaolin even with a 1:100 urine dilution and an approximate daily urine volume of 1 litre this method covers a daily hormone excretion of up to $1,000,000$ i.u.

Whenever possible an accurately collected 24-hour urine sample (preserved with chloroform) should be obtained. The expected accuracy of these titrations may not be better than $\pm 25 \%$ but this is usually adequate for clinical purposes.

The additional protein precipitating step and the smaller volume of available material makes quantitative recoveries from serum less favourable. An eight to tenfold increase in concentration per millilitre compared with that in urine is necessary for an unequivocal 'positive' result. The use of serum also requires a large venepuncture $(20 \mathrm{ml}$. of blood) and its processing is more time consuming. For these reasons it is not recommended for the diagnosis of borderline stages of early pregnancy, but it is available for urgent cases when no urine may be obtainable, e.g., in suspected ectopic pregnancies.

\section{CLINICAL MATERIAL}

Patients were instructed to submit all of their first morning urine after restricting their fluid intake for the previous 24 hours. Adherence to these instructions, however, was not checked. The tests were run in parallel with Galli-Mainini toad tests. The result of the toad tests were not known until after the results of the immunochemical tests were recorded.

The Galli-Mainini tests were performed routinely by precipitating H.C.G. from $30 \mathrm{ml}$. of chilled filtered urine by the addition of $60 \mathrm{ml}$. of chilled acetone. The centrifuged precipitate was resuspended in $3 \mathrm{ml}$. of $M / 15$

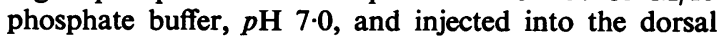
lymph sac of male Bufo marinus toads. The toads were rested for three weeks after each injection and were prescreened for sperm before injections. Toad urine was examined one to three hours after each injection, and if free from sperm, a second toad was injected with similarly prepared material. In some instances a third toad was injected with material obtained from $60 \mathrm{ml}$. of urine. This was only done with samples giving a positive immunochemical test but a negative routine GalliMainini test (discrepancy 1 in Table 1). All toads were observed repeatedly for spermiation up to 24 hours after injections. Occasional dead sperm found in one toad only was recorded as doubtful and a third toad injected. Live sperm regardless of numbers was recorded as positive. 
Serum Galli-Mainini tests were performed by injecting $3 \mathrm{ml}$. of untreated serum using similar criteria for the number of animals used.

Occasional departures from the above were due to insufficient volume of specimens submitted or the death of animals while under test. Further specimens were requested in these instances.

\section{RESULTS}

A comparison of results is summarized in the following table:

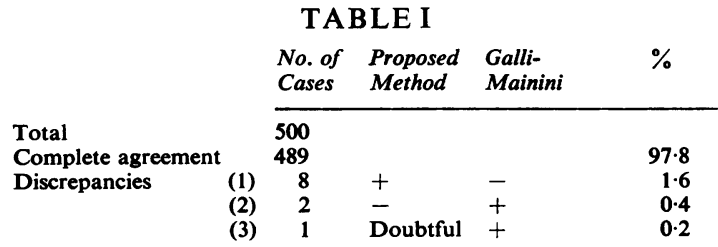

Of the eight patients shown under (1) all were proven to have been pregnant either clinically or by repeated testing of $60 \mathrm{ml}$. urine precipitates or further samples until the Galli-Mainini test also showed a positive result. Two patients were tested more than one additional time. These latter additional tests are not included in Table $I$ as they introduce a bias in favour of the greater sensitivity of the proposed test. Of these two patients one submitted four samples in all, three of which showed a positive immunochemical test with a negative Galli-Mainini and the final specimen was negative by both tests. The patient was a known aborter and in this pregnancy had aborted a few days after the last test at about 11 weeks of gestation despite supportive 'depot' therapy with progestational steroids. The other patient had submitted sufficient urine to repeat both tests on the same sample with similar results. Two days later a fresh sample gave positive results by both tests.

Of the two cases shown under (2), one specimen showed extreme bacterial contamination and a very high mucus content so that it was impossible to emulsify the kaolin completely with the method of tapping the tube. The same specimen was reprocessed by suspending the kaolin with the aid of a glass rod and gave a positive result. The reprocessing, however, was done after the Galli-Mainini results were read, so it is recorded in the Table as a 'false negative'. The other specimen also showed heavy bacterial contamination and caused spermiation of the animals only after more than 15 hours.
The one case under (3) showed very fine agglutin- $\overrightarrow{=}$

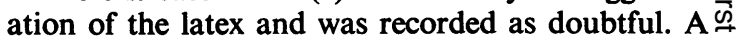
fresh specimen two days later was positive by both을 tests.

The overall number of positive and negative $\frac{\bar{p}}{\vec{\sigma}}$ results was very close to being equal in frequency so $\propto$ that the discrepancies are not biased.

It should be pointed out that these results are only comparisons between the two methods and do not suggest degrees of accuracy. 'False' negatives $\vec{\omega}$ will occur if urines are submitted too early in pregnancy when the quantities of H.C.G. are? insufficient for detection by either method. Con- $\vec{v}$ versely, it is the author's firm conviction that ir adequately prescreened toads will not give a 'false' $r$ positive Galli-Mainini test. It was therefore import- $\infty$ ant to prove that the immunochemical test 'positives'음 are equally reliable.

The number of sera tested were too few for $\mathbb{D}$ conclusions to be drawn. The impression gained, $\frac{\mathbb{T}}{\mathbb{D}}$ however, suggests that the Galli-Mainini test is the $\frac{}{3}$ more reliable for early diagnosis (Goldin, 1962).

\section{DISCUSSION}

In selecting the methods proposed the primary aim was adequate concentration and purification of $\frac{D}{0}$ the hormone together with sufficient simplicity of technique for routine use. The method of kaolin $\frac{\mathbb{}}{\square}$ adsorption alone proposed by Scott (1940) and used $\overrightarrow{\vec{A}}$ recently for complement-fixation procedures by 3 Brody and Carlström (1960) was found suitable for urine processing.

The method for serum was taken from the first steps of the technique of Rimington and Rowlands? (1951) for extracting pregnant mare serum gonadotrophin. It was mainly chosen because it lends itself to continuation with the routine urine procedure. Its author claimed a $95 \%$ recovery after the first steps of his protein precipitation. This has not been음 checked.

Several authors (Butt, Crooke, and Cunningham, 1961; Wide and Gemzell, 1962) have shown that N the rabbit antiserum to H.C.G. cross reacts with pituitary gonadotrophins. The occurrence of false $\mathcal{N}$ positives with the present method from this cause is $\mathrm{C}$ reduced on purely quantitative grounds. A two to 10-fold increase in the daily output of these gonadoe trophins would be required over the known daily excretion level in menopausal women to give fully? positive tests with the proposed procedure, but the following additional precautionary steps may be용 taken.

If the period of amenorrhoea is known to exceed seven weeks, the amount of alkaline eluent may beo 
increased from $0.1 \mathrm{ml}$. (step 3 ) to $0.3 \mathrm{ml}$. and the result interpreted in the usual manner. If the period of amenorrhoea is unknown or less than seven weeks and the result of the routine procedure is doubtful or positive in patients aged 35 years or older, 1 drop of final eluate is diluted with 2 drops of alkaline eluent and the mixture is retested. If this dilution is also positive pregnancy may be assumed. If this dilution is negative the results are reported as doubtful and a further specimen is tested one week later. If on that sample the diluted eluate is again negative the patient is menopausal and not pregnant.

With regard to economy in time and reagents, an average batch of 10 to 12 urines can be processed in one hour excluding refrigeration and clearing time. Eighteen urines, the highest number handled simultaneously in the present series were completed in one hour and 20 minutes. Well over 100 tests can be performed with one reagent kit.

\section{REFERENCES}

Brody, S., and Carlström, G. (1960). Lancet, 2, 99.

Butt, W. R., Crooke, A. C., and Cunningham, F. J. (1961). Proc. roy. Soc. Med., 54, 647 .

Goldin, M. (1962). Amer. J. clin. Path., 38, 335.

Rimington, C., and Rowlands, I. W. (1941). Biochem. J., 35, 736.

Scott, L. D. (1940). Brit. J. exp. Path., 21, 320.

Wide, L., and Gemzell, C. (1962). Acta Endocrinologica, 39, 539.

\section{ADDENDUM}

While this work was in press the Ortho Pharmaceutical Company withdrew the diagnostic reagents from the market and replaced them with a new kit now sold under the trade name of Gravindex. The immunological principle of the new kit is similar to that of the previous reagents but the performance of the test is now carried out on glass slides and is thus very much less time consuming. Preliminary investigations have shown certain disadvantages in its proposed use. Macroscopic examination of agglutination or otherwise of the added latex is too often equivocal and the amount of HCG required for full positives is about five or six times (Watson and Bettinger, 1964) greater than the accepted level for diagnosing pregnancy as suggested in the present study as well as in the haemagglutination-inhibition methods now in current use (Pregnosticon, Organon; U.C.G., Denver etc.) However, it is possible to dilute the antiserum about six-fold with a diluent capable of specifically enhancing agglutination of the latex, and examining microscopically. This yields very many fewer 'doubtfuls' and is equivalent to 1 i.u./ml. H.C.G. when mixed with equal parts of urine.

\section{REFERENCE}

Watson, D., and Bettinger, H. F. (1964). Med. J. Aust 1, 796. 